which are close to identity // Ann. Acad. Sci. Fenn. Ser. AI Math. 1968. Vol. 435. P. 3-26.

9. Прохоров Д. В., Гордиенко В. Г. Определение границы в локальной гипотезе Хажинского-Тамми // Изв. вузов. Математика. 2008. № 9. С. 59-68.

10. Прохоров Д. В. Множества значений систем функ- ционалов в классах однолистных функций // Мат. сб. 1990. Т. 181, № 12. С. 1659-1677.

11. Понтрягин Л. С., Болтянский В. Г., Гамкрелидзе Р. В., Мищенко Е. Ф. Математическая теория оптимальных процессов. М. : Наука, 1969. 384 с.

\title{
Determination of the Boundary in the Local Charzynski-Tammi Conjecture for the Fifth Coefficient
}

\section{G. Gordienko, K. A. Samsonova}

Saratov State University, Russia, 410012, Saratov, Astrahanskaya st., 83, valeriygor@mail.ru, kris-ruzhik@mail.ru

In this article we find the exact value of $M_{5}$ such that the symmetrized Pick function $P_{M 4}(z)$ is an extreme in the local CharzynskiTammi conjecture for the fifth Taylor coefficient of the normalized holomorphic bounded univalent functions

Key words: Löwner equation, optimum control, Pontryagin maxsimum principle.

\section{References}

1. Branges L. A proof of the Bieberbach conjecture. LOMI Preprints E-5-84, 1984, pp. 1-21.

2. Branges L. A proof of the Bieberbach conjecture. Acta Math., 1985, vol. 154, no 1-2, pp. 137-152.

3. Pick G. Über die konforme Abbildung eines Kreises auf ein schlichtes und zugleich beschränktes Gebiet. S.-B. Kaiserl. Akad. Wiss. Wien. Math., Naturwiss. Kl. Abt. II a, 1917, B. 126, pp. 247-263.

4. Schaeffer A. C., Spencer D. C. The coefficients of schlicht functions. Duke Math. J., 1945, vol. 12, pp. 107125.

5. Schiffer M., Tammi O. On the fourth coefficient of bounded univalent functions. Trans. Amer. Math. Soc., 1965, vol. 119 , pp. 67-78.

6. Siewierski L. Sharp estimation of the coefficients of bounded univalent functions near the identity. Bull. Acad. Polon. Sci., 1968, vol. 16, pp. 575-576.

7. Siewierski L. Sharp estimation of the coefficients of bounded univalent functions close to identity. Dissertationes Math. (Rozprawy Mat.), 1971, vol. 86, pp. 1-153.

8. Schiffer M., Tammi O. On bounded univalent functions which are close to identity. Ann. Acad. Sci. Fenn. Ser. AI Math., 1968, vol. 435, pp. 3-26.

9. Prokhorov D. V., Gordienko V. G. Definition of the boundary in the local Charzynski-Tammi conjecture. Russ. Math. (Izvestiya VUZ. Matematika), 2008, vol. 52, no. 9, pp. 51-59.

10. Prokhorov D. V. Sets of values of systems of functionals in classes of univalent functions. Mathematics of the USSR-Sbornik, 1992, vol. 71, no. 2, pp. 499-516. 11. Pontryagin L. S., Boltyanskii V. G., Gamkrelidze R. V., Mischenko E. F. Matematicheskaya teoriya optimal'nykh protsessov [The Mathematical Theory of Optimal Processes], Moscow, Nauka, 1969, 384 p. (in Russian).

УДК 517.984

\section{АНАЛОГ ТЕОРЕМЫ ЖОРДАНА-ДИРИХЛЕ ДЛЯ ИНТЕГРАЛЬНОГО ОПЕРАТОРА С ЯДРОМ, ИМЕЮЩИМ СКАЧКИ НА ЛОМАНЫХ ЛИНИЯХ}

\footnotetext{
О. А. Королева

Старший преподаватель кафредры компьютерной алгебры и теории чисел, Саратовский государственный университет им. Н. Г. Чернышевского, korolevaoart@yandex.ru

Найдены достаточные условия (условия типа Жордана-Дирихле) разложения функции $f(x)$ в равномерно сходящийся ряд по собственным и присоединенным функциям интегрального оператора, ядро которого терпит скачки на сторонах квадрата, вписанного в единичный квадрат. Как известно, для такого разложения необходимо, чтобы $f(x)$ была непрерывна и принадлежала замыканию области значений интегрального оператора. Оказывается, если $f(x)$ к тому же фрункция ограниченной вариации, эти условия являются и достаточными.

Ключевые слова: теорема Жордана-Дирихле, резольвента, характеристические числа, собственные и присоединенные фрункции.
} 
Рассмотрим интегральный оператор:

$$
y=A f=\int_{0}^{1} A(x, t) f(t) d t
$$

Обозначим $A_{1}(x, t)=A(x, t)$, если $\{0 \leq t \leq 1 / 2-x, 0 \leq x \leq 1 / 2\}, A_{2}(x, t)=A(x, t)$, если $\{1 / 2+x \leq$ $\leq t \leq 1,0 \leq x \leq 1 / 2\}, A_{3}(x, t)=A(x, t)$, если $\{0 \leq t \leq-1 / 2+x, 1 / 2 \leq x \leq 1\}, A_{4}(x, t)=A(x, t)$, если $\{3 / 2-x \leq t \leq 1,1 / 2 \leq x \leq 1\}, A_{5}(x, t)=A(x, t)$, если $\{1 / 2-x \leq t \leq 1 / 2+x, 0 \leq x \leq 1 / 2\}$ и $\{-1 / 2+x \leq t \leq 3 / 2-x, 1 / 2 \leq x \leq 1\}$.

Предположим, что $A_{i}(x, t), i=1, \ldots, 5$ непрерывно-дифференцируемые в своих областях, причем $A_{5}(x, 1 / 2-x+0)-A_{1}(x, 1 / 2-x-0)=a, A_{5}(x, 1 / 2+x-0)-A_{2}(x, 1 / 2+x+0)=b, A_{5}(x,-1 / 2+x+0)-$ - $A_{3}(x,-1 / 2+x-0)=c, A_{5}(x, 3 / 2-x-0)-A_{4}(x, 3 / 2-x+0)=d$, где $a, b, c, d$ - постоянные.

Частный случай оператора (1) впервые рассматривался в статье [1].

Рассмотрим следующий оператор:

$$
z=B g=\int_{0}^{1 / 2} B(x, t) g(t) d t, \quad 0 \leq x \leq 1 / 2,
$$

где $z(x)=\left(z_{1}(x), z_{2}(x), z_{3}(x), z_{4}(x)\right)^{T}, g(x)=\left(g_{1}(x), g_{2}(x), g_{3}(x), g_{4}(x)\right)^{T}$,

$$
B(x, t)=\left(\begin{array}{cccc}
0 & A(x, 1 / 2-t) & A(x, 1 / 2+t) & 0 \\
A(1 / 2-x, t) & 0 & 0 & A(1 / 2-x, 1-t) \\
A(1 / 2+x, t) & 0 & 0 & A(1 / 2+x, 1-t) \\
0 & A(1-x, 1 / 2-t) & A(1-x, 1 / 2+t) & 0
\end{array}\right) .
$$

Теорема 1. Если $y=A f$, то $z=B g$, где $z_{1}(x)=y(x), z_{2}(x)=y(1 / 2-x), z_{3}(x)=y(1 / 2+x)$, $z_{4}(x)=y(1-x), g_{1}(x)=f(x), g_{2}(x)=f(1 / 2-x), g_{3}(x)=f(1 / 2+x), g_{4}(x)=f(1-x)$. Обратно, если $z=$ Вg и $g_{1}(x)=g_{2}(1 / 2-x), g_{3}(x)=g_{4}(1 / 2-x)$, то $z_{1}(x)=z_{2}(1 / 2-x), z_{3}(x)=z_{4}(1 / 2-x)$ u $y=A f$, где $f(x)=g_{1}(x)$, при $x \in[0,1 / 2] ; f(x)=g_{3}(-1 / 2+x)$, при $x \in[1 / 2,1]$ и $y(x)=z_{1}(x)$, при $x \in[0,1 / 2] ; y(x)=z_{3}(-1 / 2+x)$, при $x \in[1 / 2,1]$.

Доказательство представлено в [2].

Замечание. Представление типа (2) не единственно. Наше же представление хорошо тем, что компоненты матрицы $B(x, t)$ терпят разрывы лишь на линии $t=x$.

В статье [2] также найдены необходимые и достаточные условия существования оператора $B^{-1}$. В дальнейшем будем предполагать, что $B^{-1}$ существует.

Теорема 2. Для оператора $B^{-1}$ справедливо представление

$$
\begin{gathered}
B^{-1} z(x)=P z^{\prime}(x)+a_{1}(x) z(0)+a_{2}(x) z(1 / 2)+a_{3}(x) z(x)+\int_{0}^{1 / 2} a(x, t) z(t) d t, \\
S z(0)+T z(1 / 2)+\int_{0}^{1 / 2} a(t) z(t) d t=0 .
\end{gathered}
$$

где $a_{i}(x), i=\overline{1,3}, a_{3}^{\prime}(x)$, а $(x)$ - непрерывные матрищь-функции, каждая компонента матриць $a(x, t)$ имеет такой же характер гладкости, ито и компоненты $B_{x}(x, t), S=E+\int_{0}^{1 / 2} B(0, t) a_{1}(t) d t$, $T=\int_{0}^{1 / 2} B(0, t) a_{2}(t) d t-$ постоянные матрищы $4 \times 4$.

Доказательство повторяет доказательство теоремы 10 в статье [1].

1. Получим интегродифференциальную систему для резольвенты $R_{\lambda}=(E-\lambda A)^{-1} A$ оператора А. Пусть $z=(E-\lambda B)^{-1} B g$. Тогда $z-\lambda B z=B g$. Отсюда по теореме 2 из (3), (4) получаем:

$$
P z^{\prime}(x)+a_{1}(x) z(0)+a_{2}(x) z(1 / 2)+a_{3}(x) z(x)+\tilde{N} z-\lambda z(x)=g(x),
$$




$$
S z(0)+T z(1 / 2)+\int_{0}^{1 / 2} a(t) z(t) d t=0,
$$

где $\tilde{N} z=\int_{0}^{1 / 2} a(x, t) z(t) d t$.

Теорема 3. Eсли $R_{\lambda}$ существует, то $R_{\lambda} f=v(x)$, где

$$
v(x)=z_{1}(x) \quad \text { nрu } \quad x \in[0,1 / 2] \quad u \quad v(x)=z_{3}(x-1 / 2) \quad \text { nрu } \quad x \in[1 / 2,1],
$$

$z_{1}, z_{3}$ - первая и третья компоненты вектора $z(x)$, удовлетворяющего системе (5), (6). Обратно, если $\lambda$ таково, что однородная краевая задача для (5), (6) имеет только нулевое решение, то $R_{\lambda}$ существует и определяется по формуле (7).

Доказательство повторяет лемму 1 из статьи [3].

Рассмотрим систему (5), (6). Минимальный многочлен матрицы $Q=P^{-1}$ совпадает с характеристическим многочленом и равен $\lambda^{4}-\lambda^{2}\left(d^{2}-2 b c+a^{2}\right)+(b c-a d)^{2}$. Значит, выполняется:

Лемма 1. При условии $d \neq a,(d+a)^{2}-4 b c \neq 0$ матрица $P^{-1}$ подобна диагональной $D=\operatorname{diag}\left(\omega_{1}\right.$, $\left.\omega_{2}, \omega_{3}, \omega_{4}\right)$, причём $\omega_{3}=-\omega_{2}, \omega_{4}=-\omega_{1}, \omega_{1} \neq \omega_{2}$. Пусть матрица $\Gamma$ такая, что $\Gamma^{-1} P^{-1} \Gamma=D$. Выполним в (5), (6) замену $z=$ Гг̃. Получим:

$$
\begin{gathered}
\tilde{z}^{\prime}(x)+P_{1}(x) \tilde{z}(0)+P_{2}(x) \tilde{z}(1 / 2)+P_{3}(x) \tilde{z}(x)+N \tilde{z}(x)-\lambda D \tilde{z}(x)=m(x), \\
M_{0} \Gamma \tilde{z}(0)+M_{1} \Gamma \tilde{z}(1 / 2)+\Gamma \int_{0}^{1 / 2} \Omega(t) \tilde{z}(t) d t=0,
\end{gathered}
$$

где $P_{i}(x)=D \Gamma^{-1} a_{i}(x) \Gamma, N=D \Gamma^{-1} \tilde{N} \Gamma, m(x)=D \Gamma^{-1} g(x), \Omega(t)=a(t) \Gamma, M_{0}=S \Gamma, M_{1}=T \Gamma$.

В дальнейшем при изучении системы $(8),(9)$ затруднение вызывает матрица $P_{3}(x)$. Поэтому дадим её дальнейшее преобразование.

Лемма 2. Существует матрица-функизия $H(x, \lambda)=H_{0}(x)+\lambda^{-1} H_{1}(x)$ c непрерьвнодифференцируемыми компонентами матрии $H_{0}(x), H_{1}(x)$, причем $H_{0}(x)$ невырождена при всех x и диагональная, такая, что преобразование $\tilde{z}=H(x, \lambda) v$ приводит систему (8), (9) $\kappa$ виду

$$
\begin{gathered}
v^{\prime}(x)+P_{1}(x, \lambda) v(0)+P_{2}(x, \lambda) v(1 / 2)+P_{3}(x, \lambda) v(x)+N_{\lambda} v(x)-\lambda D v(x)=m(x, \lambda), \\
U(v)=M_{0 \lambda} v(0)+M_{1 \lambda} v(1 / 2)+\int_{0}^{1 / 2} \Omega(t, \lambda) v(t) d t
\end{gathered}
$$

где $P_{1}(x, \lambda)=H^{-1}(x, \lambda) P_{1}(x) H(0, \lambda), P_{2}(x, \lambda)=H^{-1}(x, \lambda) P_{2}(x) H(1 / 2, \lambda), P_{3}(x, \lambda)=\lambda^{-1} H^{-1}(x, \lambda) \times$ $\times\left[H_{2}^{\prime}(x)+P_{3}(x) H_{2}(x)\right], N_{\lambda}=H^{-1}(x, \lambda) N H(x, \lambda), M_{0 \lambda}=M_{0} H(0, \lambda), M_{1 \lambda}=M_{1} H(1 / 2, \lambda), \Omega(t, \lambda)=$ $=\Omega(t) H(t, \lambda), m(x, t)=H^{-1}(x, \lambda) m(x)$.

Доказательство такое же, как и леммы 16 в статье [1].

Рассмотрим систему

$$
\begin{gathered}
u^{\prime}(x)=\lambda D u(x)+m(x), \\
U_{0}(u)=M_{0} H_{0}(0) u(0)+M_{1} H_{0}(1 / 2) u(1 / 2)+\int_{0}^{1 / 2} \Omega(t) H_{0}(t) u(t) d t=0,
\end{gathered}
$$

Будем считать, что $\operatorname{Re} \lambda \omega_{1} \geq \operatorname{Re} \lambda \omega_{2} \geq 0$.

Так же, как в статье [1], получаем, что для решения $u(x)$ системы $(12),(13)$ имеет место представление

$$
u(x)=u(x, \lambda)=-Y(x, \lambda) \triangle^{-1}(\lambda) \int_{0}^{1 / 2} U_{0 x}(g(x, t, \lambda)) m(t) d t+g_{\lambda} m(x),
$$

где $Y(x, \lambda)=\operatorname{diag}\left(e^{\lambda \omega_{1} x}, \ldots, e^{\lambda \omega_{4} x}\right), \triangle(\lambda)=U(Y(x, \lambda)), U_{0 x}$ означает, что $U$ применяется по $x$, $g(x, t, \lambda)=\operatorname{diag}\left(g_{1}(x, t, \lambda), \ldots, g_{4}(x, t, \lambda)\right)$,

$$
g_{i}(x, t, \lambda)= \begin{cases}-\varepsilon(t, x) e^{\lambda \omega_{i}(x-t)}, & \text { при } \operatorname{Re} \lambda \omega_{i} \geqslant 0, \\ \varepsilon(x, t) e^{\lambda \omega_{i}(x-t)}, & \text { при } \operatorname{Re} \lambda \omega_{i} \leqslant 0\end{cases}
$$




$$
\varepsilon(x, t)=\left\{\begin{array}{ll}
1, & \text { при } t \leqslant x, \\
0, & \text { при } t>x,
\end{array} \quad g_{\lambda} m(x)=\int_{0}^{1 / 2} g(x, t, \lambda) m(t) d t .\right.
$$

Лемма 3. $B S_{\delta_{0}}[2]$ при больших $|\lambda|$ компоненты матрицы $Y(x, \lambda) \triangle^{-1}(\lambda)$ имеют оценку $O(1)$ равномерно по $x \in[0,1 / 2]$.

Доказательство. Доказательство следует из оценок (27), (28) из статьи [2] для решения $u(x, \lambda)=R_{0 \lambda} m$ системы (12),(13).

Лемма 4. Имеет место

$$
\lim _{r \rightarrow \infty}\left\|\int_{|\lambda|=r}\left[H(x, \lambda) v(x, \lambda)-H_{0}(x) R_{0 \lambda}\left(H_{0}^{-1} m(x)\right)\right] d \lambda\right\|_{\infty}=0,
$$

где $v(x, \lambda)$ - решение задачи (10), (11), $\|\cdot\|_{\infty}-$ норма в $C_{\infty}$.

Доказательство аналогично доказательству леммы 11 в статье [2].

Лемма 5. При $x \in[0,1 / 2]$

$$
-\frac{1}{2 \pi i} \int_{|\lambda|=r} R_{\lambda} f d \lambda=\left(-\frac{1}{2 \pi i} \int_{|\lambda|=r} \Gamma H_{0}(x) R_{0 \lambda}\left(H_{0}^{-1} D \Gamma g\right) d \lambda\right)_{1}+o(1),
$$

где $(\cdot)_{1}-$ первая компонента вектора, о $(1) \rightarrow 0$ при $r \rightarrow \infty$ равномерно по $x \in[0,1 / 2]$.

При $x \in[1 / 2,1]$

$$
-\frac{1}{2 \pi i} \int_{|\lambda|=r} R_{\lambda} f d \lambda=\left(-\frac{1}{2 \pi i} \int_{|\lambda|=r} \Gamma H_{0}(x) R_{0 \lambda}\left(H_{0}^{-1} D \Gamma g\right) d \lambda\right)_{3}+o(1),
$$

где $(\cdot)_{3}$ - третья компонента вектора, о $(1) \rightarrow 0$ при $r \rightarrow \infty$ равномерно по $x \in[1 / 2,1]$.

Доказательство. По теореме 3 и лемме 4 аналогично теореме 4 из статьи [2].

2. Будем считать, что компоненты $m(x)$ принадлежат $C[0,1 / 2] \cap V[0,1 / 2]$. Рассмотрим подробно $R_{0 \lambda} m$ при этом условии.

Лемма 6. Имеет место формула

$$
\begin{gathered}
\int_{0}^{1 / 2} U_{0 x}(g(x, t, \lambda)) m(t) d t=-\frac{1}{\lambda} M_{0} H_{0}(0) D^{-1} g(0,1 / 2, \lambda) m(1 / 2)+\frac{1}{\lambda} M_{0} H_{0}(0) D^{-1} \times \\
\times g(0,0+, \lambda) m(0)+\frac{1}{\lambda} M_{0} H_{0}(0) D^{-1} \int_{0}^{1 / 2} g(0, t, \lambda) d m(t)-\frac{1}{\lambda} M_{1} H_{0}(1 / 2) D^{-1} g(1 / 2,1 / 2-0, \lambda) m(1 / 2)+ \\
+\frac{1}{\lambda} M_{1} H_{0}(1 / 2) D^{-1} g(1 / 2,0, \lambda) m(0)+\frac{1}{\lambda} M_{1} H_{0}(1 / 2) D^{-1} \int_{0}^{1 / 2} g(1 / 2, t, \lambda) d m(t)- \\
\quad-\frac{1}{\lambda} \int_{0}^{1 / 2} \Omega(t) H_{0}(t) D^{-1} m(t) d t+\frac{1}{\lambda} \int_{0}^{1 / 2} \Omega(\tau) H_{0}(\tau) D^{-1} d \tau \int_{0}^{1 / 2} g(\tau, t, \lambda) d m(t)+ \\
+\frac{1}{\lambda} \int_{0}^{1 / 2} \Omega(\tau) H_{0}(\tau) D^{-1} g(\tau, 0, \lambda) m(0) d \tau-\frac{1}{\lambda} \int_{0}^{1 / 2} \Omega(\tau) H_{0}(\tau) D^{-1} g(\tau, 1 / 2, \lambda) m(1 / 2) d \tau .
\end{gathered}
$$

Доказательство. В самом деле, имеем:

$$
\begin{gathered}
\int_{0}^{1 / 2} U_{0 x}(g(x, t, \lambda)) m(t) d t=M_{0} H_{0}(0) \int_{0}^{1 / 2} g(0, t, \lambda) m(t) d t+ \\
+M_{1} H_{0}(1 / 2) \int_{0}^{1 / 2} g(1 / 2, t, \lambda) m(t) d t+\int_{0}^{1 / 2} \Omega(\tau) H_{0}(\tau) d \tau \int_{0}^{1 / 2} g(\tau, t, \lambda) m(t) d t=
\end{gathered}
$$




$$
\begin{gathered}
=-\frac{1}{\lambda} M_{0} H_{0}(0) D^{-1} \int_{0}^{1 / 2} g_{t}^{\prime}(0, t, \lambda) m(t) d t-\frac{1}{\lambda} M_{1} H_{0}(1 / 2) D^{-1} \int_{0}^{1 / 2} g_{t}^{\prime}(1 / 2, t, \lambda) m(t) d t- \\
-\frac{1}{\lambda} \int_{0}^{1 / 2} \Omega(\tau) H_{0}(\tau) d \tau D^{-1} \int_{0}^{\tau} g_{t}^{\prime}(\tau, t, \lambda) m(t) d t-\frac{1}{\lambda} \int_{0}^{1 / 2} \Omega(\tau) H_{0}(\tau) d \tau D^{-1} \int_{\tau}^{1 / 2} g_{t}^{\prime}(\tau, t, \lambda) m(t) d t .
\end{gathered}
$$

Применив формулу интегрирования по частям, получим требуемое. Лемма доказана.

Рассмотрим теперь $g_{\lambda} m(x)$ из равенства (14).

Лемма 7. Справедлива формула

$$
g_{\lambda} m(x)=-\frac{1}{\lambda} D^{-1} m(x)+\frac{1}{\lambda} D^{-1} g(x, 0, \lambda) m(0)-\frac{1}{\lambda} D^{-1} g(x, 1 / 2, \lambda) m(1 / 2)+\frac{1}{\lambda} D^{-1} \int_{0}^{1 / 2} g(x, t, \lambda) d m(t) .
$$

Доказательство. Имеем:

$$
g_{\lambda} m(x)=\int_{0}^{1 / 2} g(x, t, \lambda) m(t) d t=\int_{0}^{x} g(x, t, \lambda) m(t) d t+\int_{x}^{1 / 2} g(x, t, \lambda) m(t) d t .
$$

$\mathrm{K}$ каждому интегралу применим формулу интегрирования по частям и получим требуемое. Лемма доказана.

Значит, решение системы (12), (13) принимает вид

$$
\begin{aligned}
& R_{0 \lambda} m=I_{1}+I_{2}-\frac{1}{\lambda} Y(x, \lambda) \Delta_{0}^{-1}(\lambda) M_{0} H_{0}(0) D^{-1} \int_{0}^{1 / 2} g(0, t, \lambda) d m(t)-\frac{1}{\lambda} Y(x, \lambda) \Delta_{0}^{-1}(\lambda) \times \\
& \times M_{1} H_{0}(1 / 2) D^{-1} \int_{0}^{1 / 2} g(1 / 2, t, \lambda) d m(t)-\frac{1}{\lambda} Y(x, \lambda) \Delta_{0}^{-1}(\lambda) \int_{0}^{1 / 2} \Omega(t) H_{0}(t) D^{-1} m(t) d t- \\
& -\frac{1}{\lambda} Y(x, \lambda) \Delta_{0}^{-1}(\lambda) \int_{0}^{1 / 2} \Omega(\tau) H_{0}(\tau) D^{-1} d \tau \int_{0}^{1 / 2} g(\tau, t, \lambda) d m(t)-\frac{1}{\lambda} Y(x, \lambda) \Delta_{0}^{-1} \int_{0}^{1 / 2} \Omega(\tau) \times \\
& \times H_{0}(\tau) D^{-1} g(\tau, 0, \lambda) m(0) d \tau+\frac{1}{\lambda} Y(x, \lambda) \Delta_{0}^{-1} \int_{0}^{1 / 2} \Omega(\tau) H_{0}(\tau) D^{-1} g(\tau, 1 / 2, \lambda) m(1 / 2) d \tau- \\
& -\frac{1}{\lambda} D^{-1} m(x)+\frac{1}{\lambda} D^{-1} g(x, 0, \lambda) m(0)-\frac{1}{\lambda} D^{-1} g(x, 1 / 2, \lambda) m(1 / 2)+\frac{1}{\lambda} D^{-1} \int_{0}^{1 / 2} g(x, t, \lambda) d m(t),
\end{aligned}
$$

где

$$
\begin{gathered}
I_{1}=\frac{1}{\lambda} Y(x, \lambda) \Delta_{0}^{-1} M_{0} H_{0}(0) D^{-1} g(0,1 / 2, \lambda) m(1 / 2)+ \\
+\frac{1}{\lambda} Y(x, \lambda) \Delta_{0}^{-1} M_{1} H_{0}(1 / 2) D^{-1} g(1 / 2,1 / 2-0, \lambda) m(1 / 2)-\frac{1}{\lambda} D^{-1} g(x, 1 / 2, \lambda) m(1 / 2), \\
I_{2}=-\frac{1}{\lambda} Y(x, \lambda) \Delta_{0}^{-1} M_{0} H_{0}(0) D^{-1} g(0,0+, \lambda) m(0)- \\
-\frac{1}{\lambda} Y(x, \lambda) \Delta_{0}^{-1} M_{1} H_{0}(1 / 2) D^{-1} g(1 / 2,0, \lambda) m(0)+\frac{1}{\lambda} D^{-1} g(x, 0, \lambda) m(0) .
\end{gathered}
$$

Рассмотрим в (15) некоторые слагаемые.

Лемма 8. Имеет место формула

$$
I_{1}=\frac{1}{\lambda} Y(x, \lambda) \Delta_{0}^{-1} M_{1} H_{0}(1 / 2) D^{-1} m(1 / 2)-\frac{1}{\lambda} Y(x, \lambda) \Delta_{0}^{-1} \int_{0}^{1 / 2} \Omega(t) H_{0}(t) \times
$$




$$
\times Y(t, \lambda) Y^{-1}(x, \lambda) D^{-1} g(x, 1 / 2, \lambda) d t m(1 / 2) .
$$

Доказательство. В самом деле, за счет перестановочности диагональных матриц

$$
I_{1}=\frac{1}{\lambda} Y(x, \lambda) \Delta_{0}^{-1} I D^{-1} m(1 / 2),
$$

где $I=M_{0} H_{0}(0) g(0,1 / 2, \lambda)+M_{1} H_{0}(1 / 2) g(1 / 2,1 / 2-0, \lambda)-\Delta_{0} Y^{-1}(x, \lambda) g(x, 1 / 2, \lambda)$.

Рассмотрим подробно $I$ :

$$
\begin{gathered}
I=M_{0} H_{0}(0) \operatorname{diag}\left(-e^{-\lambda \omega_{1} 1 / 2},-e^{-\lambda \omega_{2} 1 / 2}, 0,0\right)+M_{1} H_{0}(1 / 2) \operatorname{diag}(0,0,1,1)- \\
-M_{0} H_{0}(0) Y(0, \lambda) \operatorname{diag}\left(e^{-\lambda \omega_{1} x}, e^{-\lambda \omega_{2} x}, e^{-\lambda \omega_{3} x}, e^{-\lambda \omega_{4} x}\right) \operatorname{diag}\left(-e^{\lambda \omega_{1}(x-1 / 2)},-e^{\lambda \omega_{2}(x-1 / 2)}, 0,0\right)- \\
-M_{1} H_{0}(1 / 2) Y(1 / 2, \lambda) \operatorname{diag}\left(e^{-\lambda \omega_{1} x}, e^{-\lambda \omega_{2} x}, e^{-\lambda \omega_{3} x}, e^{-\lambda \omega_{4} x}\right) \operatorname{diag}\left(-e^{\lambda \omega_{1}(x-1 / 2)},-e^{\lambda \omega_{2}(x-1 / 2)}, 0,0\right)- \\
-\int_{0}^{1 / 2} \Omega(t) H_{0}(t) Y(t, \lambda) Y^{-1}(x, \lambda) g(x, 1 / 2, \lambda) d t=M_{0} H_{0}(0)\left[\operatorname{diag}\left(-e^{-\lambda \omega_{1} 1 / 2},-e^{-\lambda \omega_{2} 1 / 2}, 0,0\right)+\right. \\
\left.+\operatorname{diag}(1,1,1,1) \operatorname{diag}\left(e^{-\lambda \omega_{1} x}, e^{-\lambda \omega_{2} x}, e^{-\lambda \omega_{3} x}, e^{-\lambda \omega_{4} x}\right) \operatorname{diag}\left(e^{\lambda \omega_{1}(x-1 / 2)}, e^{\lambda \omega_{2}(x-1 / 2)}, 0,0\right)\right]+ \\
+M_{1} H_{0}(1 / 2)\left[\operatorname{diag}(0,0,1,1)+\operatorname{diag}\left(e^{\lambda \omega_{1} 1 / 2}, e^{\lambda \omega_{2} 1 / 2}, e^{\lambda \omega_{3} 1 / 2}, e^{\lambda \omega_{4} 1 / 2}\right) \times\right. \\
\left.\quad \times \operatorname{diag}\left(e^{-\lambda \omega_{1} x}, e^{-\lambda \omega_{2} x}, e^{-\lambda \omega_{3} x}, e^{-\lambda \omega_{4} x}\right) \operatorname{diag}\left(e^{\lambda \omega_{1}(x-1 / 2)}, e^{\lambda \omega_{2}(x-1 / 2)}, 0,0\right)\right]- \\
-\int_{0}^{1 / 2} \Omega(t) H_{0}(t) Y(t, \lambda) Y^{-1}(x, \lambda) g(x, 1 / 2, \lambda) d t=M_{0} H_{0}(0)\left[\operatorname{diag}\left(-e^{-\lambda \omega_{1} / 2},-e^{-\lambda \omega_{2} / 2}, 0,0\right)+\right. \\
\left.+\operatorname{diag}\left(e^{-\lambda \omega_{1} / 2}, e^{-\lambda \omega_{2} / 2}, 0,0\right)\right]+M_{1} H_{0}(1 / 2)[\operatorname{diag}(0,0,1,1)+\operatorname{diag}(1,1,0,0)]- \\
-\int_{0}^{1 / 2} \Omega(t) H_{0}(t) Y(t, \lambda) Y^{-1}(x, \lambda) g(x, 1 / 2, \lambda) d t .
\end{gathered}
$$

Первое слагаемое обращается в ноль, и мы получили требуемое. Лемма доказана.

Лемма 9. Имеет место формула

$$
\begin{aligned}
I_{2}=\frac{1}{\lambda} Y(x, \lambda) & \Delta_{0}^{-1} M_{0} H_{0}(0) D^{-1} m(0)+\frac{1}{\lambda} Y(x, \lambda) \Delta_{0}^{-1} \int_{0}^{1 / 2} \Omega(t) H_{0}(t) \times \\
& \times Y(t, \lambda) Y^{-1}(x, \lambda) D^{-1} g(x, 0, \lambda) d t m(0) .
\end{aligned}
$$

Доказательство аналогично доказательству леммы 8.

Значит равенство (15) приобретает вид

$$
\begin{aligned}
R_{0 \lambda} m & =\frac{1}{\lambda} Y(x, \lambda) \Delta_{0}^{-1} M_{0} H_{0}(0) D^{-1} m(0)+\frac{1}{\lambda} Y(x, \lambda) \Delta_{0}^{-1} M_{1} H_{0}(1 / 2) D^{-1} m(1 / 2)+\frac{1}{\lambda} Y(x, \lambda) \Delta_{0}^{-1} \times \\
& \times \int_{0}^{1 / 2} \Omega(t) H_{0}(t) D^{-1} m(t) d t-\frac{1}{\lambda} Y(x, \lambda) \Delta_{0}^{-1} M_{0} H_{0}(0) D^{-1} \int_{0}^{1 / 2} \Omega(t) H_{0}(t) g(0, t, \lambda) d m(t)- \\
& -\frac{1}{\lambda} Y(x, \lambda) \Delta_{0}^{-1} M_{1} H_{0}(1 / 2) D^{-1} \int_{0}^{1 / 2} \Omega(t) H_{0}(t) g(1 / 2, t, \lambda) d m(t)-\frac{1}{\lambda} Y(x, \lambda) \Delta_{0}^{-1} \int_{0}^{1 / 2} \Omega(\tau) \times \\
& \times H_{0}(\tau) d \tau D^{-1} \int_{0}^{1 / 2} g(\tau, t, \lambda) d m(t)+\frac{1}{\lambda} D^{-1} \int_{0}^{1 / 2} g(x, t, \lambda) d m(t)-\frac{1}{\lambda} Y(x, \lambda) \Delta_{0}^{-1}\left[\tilde{I}_{1}+\tilde{I}_{2}\right],
\end{aligned}
$$

где

$$
\tilde{I}_{1}=\int_{0}^{1 / 2} \Omega(t) H_{0}(t) Y(t, \lambda) Y^{-1}(x, \lambda) g(x, 1 / 2, \lambda) d t D^{-1} m(1 / 2)-\int_{0}^{1 / 2} \Omega(t) H_{0}(t) g(t, 1 / 2, \lambda) d t D^{-1} m(1 / 2),
$$




$$
\tilde{I}_{2}=-\int_{0}^{1 / 2} \Omega(t) H_{0}(t) Y(t, \lambda) Y^{-1}(x, \lambda) g(x, 0, \lambda) d t D^{-1} m(0)+\int_{0}^{1 / 2} \Omega(t) H_{0}(t) g(t, 0, \lambda) d t D^{-1} m(0) .
$$

Лемма 10. В равенстве (16) $\tilde{I}_{1}=\tilde{I}_{2}=0$.

Доказательство. Рассмотрим, например, $\tilde{I}_{1}$ :

$$
\tilde{I}_{1}=\int_{0}^{1 / 2} \Omega(t) H_{0}(t) I d t D^{-1} m(1 / 2),
$$

где $I=Y(t, \lambda) Y^{-1}(x, \lambda) g(x, 1 / 2, \lambda)-g(t, 1 / 2, \lambda)$. В свою очередь,

$$
\begin{gathered}
I=\operatorname{diag}\left(e^{\lambda \omega_{1} t}, e^{\lambda \omega_{2} t}, e^{\lambda \omega_{3} t}, e^{\lambda \omega_{4} t}\right) \cdot \operatorname{diag}\left(e^{-\lambda \omega_{1} x}, e^{-\lambda \omega_{2} x}, e^{-\lambda \omega_{3} x}, e^{-\lambda \omega_{4} x}\right) \times \\
\times \operatorname{diag}\left(-e^{\lambda \omega_{1}(x-1 / 2)},-e^{\lambda \omega_{2}(x-1 / 2)}, 0,0\right)-\operatorname{diag}\left(-e^{\lambda \omega_{1}(t-1 / 2)},-e^{\lambda \omega_{2}(t-1 / 2)}, 0,0\right)= \\
=\operatorname{diag}\left(-e^{\lambda \omega_{1}(t-1 / 2)},-e^{\lambda \omega_{2}(t-1 / 2)}, 0,0\right)+\operatorname{diag}\left(e^{\lambda \omega_{1}(t-1 / 2)}, e^{\lambda \omega_{2}(t-1 / 2)}, 0,0\right)=0 .
\end{gathered}
$$

Аналогично равенство устанавливается и для $\tilde{I}_{2}$. Лемма доказана.

Равенство (16) теперь приобретает вид

$$
R_{0 \lambda} m=\frac{1}{\lambda} Y(x, \lambda) \Delta_{0}^{-1} J-\frac{1}{\lambda} D^{-1} m(x)+\Omega_{\lambda} m(x),
$$

где

$$
\begin{gathered}
J=M_{0} H_{0}(0) D^{-1} m(0)+M_{1} H_{0}(1 / 2) D^{-1} m(1 / 2)+\int_{0}^{1 / 2} \Omega(t) H_{0}(t) D^{-1} m(t) d t, \\
\Omega_{\lambda} m(x)=-\frac{1}{\lambda} Y(x, \lambda) \Delta_{0}^{-1} M_{0} H_{0}(0) D^{-1} \int_{0}^{1 / 2} \Omega(t) H_{0}(t) g(0, t, \lambda) d m(t)- \\
-\frac{1}{\lambda} Y(x, \lambda) \Delta_{0}^{-1} M_{1} H_{0}(1 / 2) D^{-1} \int_{0}^{1 / 2} \Omega(t) H_{0}(t) g(1 / 2, t, \lambda) d m(t)- \\
-\frac{1}{\lambda} Y(x, \lambda) \Delta_{0}^{-1} \int_{0}^{1 / 2} \Omega(\tau) H_{0}(\tau) d \tau D^{-1} \int_{0}^{1 / 2} g(\tau, t, \lambda) d m(t)+\frac{1}{\lambda} D^{-1} \int_{0}^{1 / 2} g(x, t, \lambda) d m(t) .
\end{gathered}
$$

Вернемся к лемме (5). Рассмотрим $R_{0 \lambda} m$ при $m(x)=H_{0}^{-1}(x) D \Gamma g(x)$.

Лемма 11. Если $m(x)=H_{0}^{-1}(x) D \Gamma g(x)$ и $g(x)$ удовлетворяет условию (6), то

$$
R_{0 \lambda} m=-\frac{1}{\lambda} D^{-1} m(x)+\Omega_{\lambda} m(x) .
$$

Доказательство. Рассмотрим $J$ в равенстве (17) при $m(x)=H_{0}^{-1}(x) D \Gamma g(x)$ :

$$
\begin{gathered}
J=M_{0} H_{0}(0) D^{-1} H_{0}^{-1}(0) D \Gamma g(0)+M_{1} H_{0}(1 / 2) D^{-1} H_{0}^{-1}(1 / 2) D \Gamma g(1 / 2)+ \\
+\int_{0}^{1 / 2} \Omega(t) H_{0}(t) D^{-1} H_{0}^{-1}(t) D \Gamma g(t) d t .
\end{gathered}
$$

Подставим $M_{0}=S \Gamma, M_{1}=T \Gamma, \Omega(t)=a(t) \Gamma$ из леммы (1) и воспользуемся перестановочностью диагональных матриц $D^{-1}$ и $H_{0}(x)$, получим:

$$
J=S g(0)+T g(1 / 2)+\int_{0}^{1 / 2} a(t) g(t) d t .
$$

Так как $g(x)$ удовлетворяет условию (6), то $J=0$. Лемма доказана. 
Лемма 12. $B S_{\delta_{0}}$ при больших $|\lambda|$

$$
\left\|\int_{|\lambda|=r}\left(\Omega_{\lambda} m\right)(x) d \lambda\right\|_{\infty} \underset{r \rightarrow \infty}{\longrightarrow} 0 .
$$

Доказательство. Имеем:

$$
\Omega_{\lambda} m(x)=I_{1}+I_{2}+I_{3}+I_{4}
$$

где теперь

$$
\begin{gathered}
I_{1}=-\frac{1}{\lambda} Y(x, \lambda) \Delta_{0}^{-1} M_{0} H_{0}(0) D^{-1} \int_{0}^{1 / 2} \Omega(t) H_{0}(t) g(0, t, \lambda) d m(t), \\
I_{2}=-\frac{1}{\lambda} Y(x, \lambda) \Delta_{0}^{-1} M_{1} H_{0}(1 / 2) D^{-1} \int_{0}^{1 / 2} \Omega(t) H_{0}(t) g(1 / 2, t, \lambda) d m(t), \\
I_{3}=-\frac{1}{\lambda} Y(x, \lambda) \Delta_{0}^{-1} \int_{0}^{1 / 2} \Omega(\tau) H_{0}(\tau) d \tau D^{-1} \int_{0}^{1 / 2} g(\tau, t, \lambda) d m(t), \quad I_{4}=\frac{1}{\lambda} D^{-1} \int_{0}^{1 / 2} g(x, t, \lambda) d m(t) .
\end{gathered}
$$

Рассмотрим первое слагаемое:

$$
\begin{aligned}
I_{1}=-\frac{1}{\lambda} Y(x, \lambda) \Delta_{0}^{-1} & M_{0} H_{0}(0) D^{-1} \int_{0}^{1 / 2} \operatorname{diag}\left(-e^{-\lambda \omega_{1} t},-e^{-\lambda \omega_{2} t}, 0,0\right) d m(t)=-\frac{1}{\lambda} Y(x, \lambda) \Delta_{0}^{-1} \times \\
& \times M_{0} H_{0}(0) D^{-1} \int_{0}^{1 / 2}\left(-e^{-\lambda \omega_{1} t} d m_{1},-e^{-\lambda \omega_{2} t} d m_{2}, 0,0\right)^{T} .
\end{aligned}
$$

Так как (лемма 3) компоненты матрицы $Y(x, \lambda) \Delta_{0}^{-1}$ имеют оценку $O(1)$, то после перемножения компоненты вектора $I_{1}$ имеют вид

$$
J_{1}+J_{2}=\frac{1}{\lambda} \int_{0}^{1 / 2} O(1) e^{-\lambda \omega_{1} t} d m_{1}+\int_{0}^{1 / 2} O(1) e^{-\lambda \omega_{2} t} d m_{2},
$$

где $O(1)-$ разные ограниченные функции. Тогда, для произвольного $x \in[0,1 / 2]$

$$
\left|J_{1}\right| \leq \frac{c}{r} \int_{0}^{1 / 2}\left|e^{-\lambda \omega_{1} t}\right|\left|d m_{1}\right| .
$$

Зададим сколь угодно малое $\varepsilon$. Тогда существует $\delta=\delta(\varepsilon)$ такое, что $\underset{0}{\bigvee_{0}}\left(m_{1}\right)<\varepsilon$. Значит,

$$
\begin{gathered}
\left|J_{1}\right| \leq \frac{c}{r} \int_{0}^{\delta}\left|e^{-\lambda \omega_{1} t}\right|\left|d m_{1}\right|+\frac{c}{r} \int_{\delta}^{1 / 2}\left|e^{-\lambda \omega_{1} t}\right|\left|d m_{1}\right| \leq \frac{c}{r} \int_{0}^{\delta}\left|d m_{1}\right|+\frac{c}{r} \int_{\delta}^{1 / 2}\left|e^{-\lambda \omega_{1} \delta}\right|\left|d m_{1}\right| \leq \\
\leq \frac{c}{r} \bigvee_{0}^{\delta}\left(m_{1}\right)+\frac{c}{r}\left|e^{-\lambda \omega_{1} \delta}\right| \bigvee_{0}^{1 / 2}\left(m_{1}\right) \leq \frac{c}{r} \varepsilon+\frac{c}{r}\left|e^{-\lambda \omega_{1} \delta}\right|,
\end{gathered}
$$

где через с обозначены разные константы. Также оценивается $J_{2}$ :

$$
\left|J_{2}\right| \leq \frac{c}{r} \varepsilon+\frac{c}{r}\left|e^{-\lambda \omega_{2} \delta}\right|
$$


Значит,

$$
\left\|I_{1}\right\|_{\infty} \leq \frac{c}{r} \varepsilon+\frac{c}{r}\left|e^{-\lambda \omega_{2} \delta}\right|
$$

Аналогично оцениваются $I_{2}, I_{3}, I_{4}$. Тогда

$$
\left\|\int_{|\lambda|=r}\left(\Omega_{\lambda} m\right)(x) d \lambda\right\|_{\infty} \leq \frac{c}{r} \varepsilon \int_{|\lambda|=r}|d \lambda|+\frac{c}{r} \int_{|\lambda|=r}\left|e^{-\lambda \omega_{2} \delta}\right||d \lambda|=\pi \varepsilon+\frac{c}{r} \underset{r \rightarrow \infty}{\longrightarrow} 0,
$$

в силу произвольности $\varepsilon$. Лемма доказана.

Теорема 4. Если $f(x) \in \bar{\Delta}_{A}$, где $\bar{\Delta}_{A}-$ замькание по норме $C[0,1]$ области значений оператоpa $A$ u $f(x) \in V[0,1]$, mo

$$
\left\|f(x)-S_{r}(f, x)\right\|_{\infty} \underset{r \rightarrow \infty}{\longrightarrow} 0 .
$$

Доказательство. Известно [4], что $\bar{\Delta}_{A}$ состоит из непрерывных функций, удовлетворяющих условию (6). Рассмотрим $S_{r}(f, x)$ По лемме (5) для $x \in[0,1 / 2]$ :

$$
S_{r}(f, x)=-\frac{1}{2 \pi i} \int_{|\lambda|=r} R_{\lambda} f d \lambda=\left(-\frac{1}{2 \pi i} \int_{|\lambda|=r} \Gamma H_{0}(x) R_{0 \lambda}\left(H_{0}^{-1} D \Gamma g\right) d \lambda\right)_{1}+o(1)
$$

По (18) имеем:

$$
\begin{gathered}
S_{r}(f, x)=\left(\frac{1}{2 \pi i} \Gamma H_{0}(x) \int_{|\lambda|=r} \frac{1}{\lambda} D^{-1} m(x) d \lambda\right)_{1}-\left(\Gamma H_{0}(x) \frac{1}{2 \pi i} \int_{|\lambda|=r} \Omega_{\lambda} m(x) d \lambda\right)_{1}+o(1)= \\
=\left(\Gamma H_{0}(x) D^{-1} m(x)\right)_{1}-\left(\Gamma H_{0}(x) \frac{1}{2 \pi i} \int_{|\lambda|=r} \Omega_{\lambda} m(x) d \lambda\right)_{1}+o(1) .
\end{gathered}
$$

Так как $m(x)=H_{0}^{-1}(x) D \Gamma g(x)$, то

$$
S_{r}(f, x)=f(x)-\left(\Gamma H_{0}(x) \frac{1}{2 \pi i} \int_{|\lambda|=r} \Omega_{\lambda} m(x) d \lambda\right)_{1}+o(1) .
$$

Для $x \in[1 / 2,1]$ получаем аналогичное равенство с $(\cdot)_{3}$ вместо $(\cdot)_{1}$. В силу леммы 12 теорема доказана.

\section{Библиографический список}

1. Хромов А. П. Интегральные операторы с ядрами, разрывными на ломаных линиях // Мат. сб. 2006. Т. 197, № 11. C 115-142. DOI: $10.4213 / \mathrm{sm} 1534$.

2. Королева О.А., Хромов А.П. Интегральный оператор с ядром, имеющим скачки на ломаных линиях // Изв. Сарат. ун-та. Нов. сер. Сер. Математика. Механика. Информатика. 2012. Т. 12, № 2. С. 6-13.

3. Корнев В.В., Хромов А. П. О равносходимости разложений по собственным функциям интегральных опера- торов с ядрами, допускающими разрывы производных на диагоналях // Мат. сб. 2001. Т. 192, № 10. С 33-50. DOI: $10.4213 / \mathrm{sm} 601$.

4. Королева О.А. О сходимости средних Рисса разложений по собственным и присоединенным функциям интегрального оператора с ядром, имеющим скачки на ломаных линиях // Изв. Сарат. ун-та. Нов. сер. Сер. Математика. Механика. Информатика. 2013. Т. 13, вып. 1, ч. 2. С. $63-67$.

\section{An Analogue of the Jordan-Dirichlet Theorem for the Integral Operator with Kernel Having Jumps on Broken Lines}

\section{O. A. Koroleva}

Saratov State University, Russia, 410012, Saratov, Astrahanskaya st., 83, korolevaoart@yandex.ru

In this paper the sufficient conditions (conditions such as Jordan-Dirichlet) expansion function $f(x)$ in a uniformly convergent series of eigenfunctions and associated functions of the integral operator whose kernel is suffering jumps on the sides of the square, inscribed in the unit square. As is known, this expansion requires to $f(x)$ is continuous and belong to the closure of the integral values operator. It turns out that if $f(x)$ also is a function of bounded variation, these conditions are also sufficient.

Key words: Jordan-Dirichlet theorem, resolvent, eigenvalues, eigenfunctions and associated functions. 


\title{
References
}

1. Khromov A. P. Integral operators with kernels that are discontinuous on broken lines. Sbornik: Mathematics, 2006, vol. 197, no. 11, pp. 1669-1696. DOI: 10.4213/sm1534.

2. Koroleva O A., Khromov A. P. Integral operator with a kernel that has jumps on broken lines. Izv. Sarat. Univ. N.S. Ser. Math. Mech. Inform., 2012, vol. 12, iss. 2, pp. 613 (in Russian).

3. Kornev V. V., Khromov A. P. Uniform convergence

of expansions in eigenfunctions of integral operators with kernels that can have discontinuities on the diagonals. Sbornik: Mathematics, 2001, vol. 192, no. 10, pp. 14511469. DOI: $10.4213 / \mathrm{sm} 601$.

4. Koroleva O A. On Convergence of Riesz Means of the Expansions in Eigen and Associated Functions Integral Operator with Kernel Having Jumps on Broken Lines. Izv. Sarat. Univ. N.S. Ser. Math. Mech. Inform., 2013, vol. 1, iss. 2, pp. 63-67 (in Russian).

\section{УДК УДК 501.1}

\section{К ЗАДАЧЕ О ЦЕЛОСТНОСТИ L-ФУНКЦИИ АРТИНА}

\section{В. Н. Кузнецов ${ }^{1}$, В. В. Кривобок ${ }^{2}$, Д. С. Степаненко ${ }^{3}$}

\begin{abstract}
${ }^{1}$ Доктор фризико-математических наук, заведующий касредрой компьютерной алгебры и теории чисел, Саратовский государственный университет им. Н. Г. Чернышевского, KuznetsovVN@info.sgu.ru

${ }^{2}$ Кандидат фризико-математических наук, доцент касредры компьютерной алгебры и теории чисел, Саратовский государственный университет им. Н. Г. Чернышевского, KrivobokVV@info.sgu.ru

${ }^{3}$ Ассистент кафредры компьютерной алгебры и теории чисел, Саратовский государственный университет им. Н. Г. Чернышевского, stepanenko.dmitry@gmail.com
\end{abstract}

В работе определяется класс $L$-срункций Артина, которые являются мероморсрными фрункциями, полюсы которых лежат на критической прямой $\operatorname{Re} s=1 / 2$ и совпадают с нулями $Z$-фрункций Дедекинда некоторых числовых полей.

Ключевые слова: $L$-фрункция Артина, теорема Брауэра.

\section{ВВЕДЕНИЕ}

Пусть $K$ - нормальное расширение числового поля $k$ степени $n$ и $G$ - группа Галуа этого расширения. Пусть $\{M(g)\}_{g \in G}-$ представление группы $G$ в группу матриц размерности $n \times n$ и $\chi-$ характер этого представления:

$$
\chi(g)=\operatorname{Sp} M(g), \quad g \in G,
$$

где $\operatorname{Sp} M(g)$ означает след матрицы $M(g)$.

$L$-функция Артина определяется следующим образом:

$$
L(s, \chi)=L(s, \chi, K \mid k)=\prod_{\wp}\left|I-M\left(\left[\frac{K \mid k}{\wp}\right]\right) N\left(\wp^{-s}\right)\right|^{-1},
$$

где ю- неразветвленный простой идеал поля $k,\left[\frac{K / k}{\wp}\right]-$ автоморфизм Фробениуса (т. е. образующий элемент, связанный с расширением классов вычетов по модулю ю), а $\left|I-M\left(\left[\frac{K / k}{\wp}\right]\right) N\left(\wp^{-s}\right)\right|-$ характеристический многочлен матрицы $M(g)$ при $\lambda=N(\wp)^{-s}$.

Отметим некоторые свойства $L$-функции Артина $[1,2]$.

1. $L(s, \chi)$ регулярна при $\sigma>1$.

2. Если расширение $K \mid k$ абелево, а $\chi$ - простой характер, то определение функции $L(s, \chi)$ за вычетом множителей, относящихся к разветвленным простым идеалам, совпадает с $L$-функцией Дирихле.

3. Пусть $\Omega$ - промежуточное поле между $K$ и $k$, являющееся нормальным над $k$. Пусть $H=\operatorname{Gal}(K \mid \Omega)$ так, что $H-$ нормальный делитель в $G$ и $G \mid H=\operatorname{Gal}(\Omega \mid k)$.

Тогда каждый характер $\chi$ группы $G \mid H$ можно очевидным образом рассматривать как характер группы $G$, причем $L(s, \chi, K \mid k)=L(s, \chi, \Omega \mid k)$.

4. Предположим, что $\chi-$ непростой характер в $G$, а именно $\chi=\chi_{1}+\chi_{2}$. Тогда $L(s, \chi)=L\left(s, \chi_{1}\right) L\left(s, \chi_{2}\right)$. 